\title{
Das Regnum Hungaricum in der Repräsentation Maria Theresias
}

Ziel des Beitrags ist es, anhand ausgewählter Themen und Kunstwerke die Frage zu erörtern, welche Rolle das Königreich Ungarn in der Repräsentation und Imagebildung Maria Theresias gespielt hat, welche Konzepte des Regnum Hungaricum im Hintergrund der reichen politischen Ikonografie Maria Theresias stehen, und welche Themen, Stereotypen, Topoi und Mythen sich in den allegorischen Darstellungen Ungarns widerspiegeln.

Die persönliche Beziehung Maria Theresias zu Ungarn war durch eine Art Sympathie charakterisiert. Sie betonte während ihrer langen Regierungszeit mehrfach ihre Zuneigung zu den Ungarn, so schrieb sie beispielsweise im November 1765 an ihren Schwiegersohn, Herzog Albert von Sachsen-Teschen, den Statthalter von Ungarn: „J'ai toujours aimé la nation hongroise, parce qu'elle est la base pour notre commun bonheur." ${ }^{175}$ („Ich habe die ungarische Nation immer geliebt; denn sie ist die Grundlage unseres gemeinsamen Glückes.") Derselbe Gedanke ist in einem Brief der Regentin gegen Ende ihres Lebens, im Juni 1778 an Herzog Albert ausgedrückt: „Je suis bonne hongroise, mon cœur est plein de reconnoisance pour cette nation. “176 („Ich bin eine gute Ungarin. Mein Herz ist voller Erkenntlichkeit gegenüber dieser Nation.") Die erste Biografie über Kaiser Joseph II. hat diesen Umstand folgendermaßen ausgedrückt: „Die immer dankbare Theresia wählte eine der glücklichsten Mittel, um der ungarischen Nation ihre Gnade und Gewogenheit recht anschaulich darzustellen. Ihr geliebter Prinz Joseph wurde in die ungarische Nationaltracht gekleidet, erhielt Unterricht in der ungarischen Sprache [...]. “177 Diese Aussprüche drücken eine Art Verbundenheit der Monarchin mit dem Königreich Ungarn aus, die zweifellos aus jenem Dankesgefühl resultierte, welches sie gegenüber der ungarischen „Nation“ hegte. Der Ausgangspunkt dieses Verhältnisses war wahrscheinlich die legendäre Szene des Preßburger Landtags am 11. September 1741, als die junge
Königin in einer lateinischen Rede um die Hilfe der ungarischen Stände gebeten hatte: „[...] Agitur de coronae istius securitate. Agitur de persona et prolibus nostris. Ideo, derelictae ab omnibus, ad arma, ad priscam virtutem et belligeram fidelitatem Hungarorum, tot historiarum monumentis celebrem, confugimus [...]." (Es gilt um die Sicherheit dieser Crone. Es gilt um unsere von allem andern Beystand verlassene Person und Kinder. Wir nehmen darum allein unsere Zuflucht zu den Waffen, zu der alten Tapferkeit und Helden-Treue der Ungarn, welche so viele Denckmale in den Geschichten berühmt gemacht haben.)“178 Gemäß dem Bericht der Zeremonialprotokolle rührte die Rede die Anwesenden zu Tränen, und die ungarischen Magnaten sicherten der Regentin mit dem Ausruf „,vitam et sanguinem dabimus pro Majestate Vestra Sacratissima“ („Unser Leben und Blut für unseren Herrscher") volle militärische Unterstützung zu. ${ }^{179}$

Am Anfang des Österreichischen Erbfolgekriegs waren die von den ungarischen Ständen bereitgestellten Truppen tatsächlich ausschlaggebend für Maria Theresias militärisches Überleben. Die grundsätzliche Bedeutung Ungarns für die Regentin am Anfang ihrer Regierung lag auch darin, dass das Regnum Hungaricum ihr den königlichen Titel verlieh, der im Rang höher stand als ihre österreichische Erzherzogswürde. Zwar stand nach der Kaiserkrönung Franz I. Stephans (1745) der Kaiserintitel an erster Stelle in der Titulatur Maria Theresias, wie es auch die Inschriften der Münzen und Medaillen der Zeit zeigen, doch bedeutete die Imperatrix-Würde für sie keinerlei konkrete zusätzliche politische Macht. Ihre höchsten tatsächlichen Herrscherwürden blieben weiterhin der ungarische und (ab 1743) der böhmische Königstitel.

Das Regnum Hungaricum nahm einen prominenten Platz nicht nur in der Titulatur Maria Theresias ein; das Land bildete einen wesentlichen Teil der Habsburgermon-

175 Wolf 1863 , Bd. $1,55$.

176 Wolf $1859,353$.

177 Johann Pezzl, Charakteristik Josephs II.: eine historisch-biographische Skizze, Wien 1790, 11.

178 Johann Daniel von Olenschlager, Geschichte des Interregni nach Absterben Kayser Karls VI., Dritter Theil, Frankfurt am Main: Varrentrapp 1744, 367.

179 HHStA, OMeA, ZP 18, fol. 345v; vgl. in diesem Band den Beitrag von Thomas Lau, \#\#. 
archie, sowohl in territorialer wie auch in demografischer Hinsicht: Die Fläche der Länder der ungarischen Krone mit Siebenbürgen betrugen etwa 325.000 Quadratkilometer, was über die Hälfte der Gesamtfläche der Habsburgermonarchie ausmachte, auch nach dem Erwerb Galiziens im Jahre 1772. ${ }^{180}$ Die Bevölkerungszahlen zeigen ähnliche Größenverhältnisse: Um 1780 machten die über neun Millionen Einwohner Ungarns und Siebenbürgens ungefähr zwei Drittel der Bevölkerung der Monarchie aus, die insgesamt also etwa 15 Millionen Einwohner hatte. ${ }^{181}$

In der sakralen Repräsentation und symbolischen Legitimierung Maria Theresias war der historische Aspekt des Königreichs Ungarn ein prägender Faktor, der insbesondere in der Verehrung des hl. Königs Stephan, des Gründers des Königreichs Ungarn, zum Ausdruck kam. Besonders die zweite Hälfte ihrer Regierungszeit war von einer Wiederbelebung des St.-Stephan-Kults geprägt, wodurch das Regnum Hungaricum als Erbe des hl. Stephan inszeniert wurde. Die Wiederaufnahme des Herrschertitels Rex Apostolicus (1758), den Papst Silvester II. im Jahr 1000 an König Stephan I. verliehen hatte, die Gründung des königlich-ungarischen St.-Stephans-Ordens (1764), die Rückgewinnung der wichtigsten Reliquie des heiligen Königs, der sogenannten Heiligen Rechten, aus Dubrovnik (Ragusa) nach Ungarn und deren Unterbringung in der Kapelle des königlichen Palastes in Buda (1771), die Erhebung des St. Stephansfestes vom 20. August zum nationalen Feiertag (1771) oder die Stiftung des silbernen Büstenreliquiars des Heiligen nach Székesfehérvár (Stuhlweißenburg) (1778) betonten Maria Theresias Rolle als würdige und legitime Nachfolgerin des Staatsgründer-Königs, da sie mit der Stephanskrone, im Krönungsmantel, bzw. in den zeremoniellen Kleidungstücken König Stephans gekrönt worden war. ${ }^{182}$ Dieser Gedanke wird oft in der Panegyrik der Zeit zum Ausdruck gebracht, so beispielsweise in der Festrede, die der Propst von
Veszprém beim Fest des hl. Stephan am 20. August 1741 in der Franziskanerkirche in Preßburg hielt, in der er zum hl. Stephan betete: „Heiliger König Stephan! Hilf deinem Successor, Königin Maria II., die im Heiligtum und in der Kraft deiner Gewänder und mit deiner heiligen englischen Krone gekrönt worden ist. “183 In der Festrede, die anlässlich der Konsekration der Kapelle des königlichen Palastes in Buda 1769 vom Dekan der Tyrnauer Universität gehalten wurde, pries dieser Maria Theresia als Bauherrin und setzte ihre Tugenden und Verdienste mit jenen des hl. Stephan, des Begründers der ungarischen Kirche, gleich. ${ }^{184}$ Die persönliche Stephansverehrung Maria Theresias manifestierte sich unter anderem darin, dass sie 1770 Pierre Joseph Verhaghens Gemälde König Stephan empfängt die Gesandten des Papstes, die ihm die Königskrone überbringen, erwarb und in ihrer Privatkapelle im Preßburger königlichen Schloss anbringen ließ. ${ }^{185}$

Die besondere Verehrung des hl. Stephan durch Maria Theresia wurde auch im Zusammenhang mit dem Gedanken des Regnum Marianum in der Panegyrik thematisiert. Nach der berühmten Legende soll König Stephan kurz vor seinem Tod Ungarn dem Schutz der hl. Jungfrau empfohlen haben. Die Gottesmutter wurde dadurch als Patrona Hungariae verehrt und das Land als Königreich Mariens verstanden, wodurch die Staatsidee des Regnum Hungaricum auch eine sakrale Dimension erhielt. ${ }^{186}$ Dieser Topos kam im 17. Jahrhundert auf Einfluss des religiös-politischen Denkens ungarischer Jesuiten in den Vorstellungen Ungarns auf und war noch in der Ära Maria Theresias sehr beliebt: Der besondere Schutz des Landes durch Maria wurde oft sowohl in Bildmedien wie auch in Schriften der Zeit thematisiert. Ein schönes Beispiel dazu ist das ehemalige Hochaltarbild der Michaelerkirche in Sopron (Ödenburg) von Bartolomeo Altomonte (1739), auf dem der Erzengel Michael und die hl. Jungfrau das Land, repräsentiert durch

180 Nach dem Verlust Schlesiens betrug die Fläche der sogenannten cisleithanischen Gebiete der Habsburgermonarchie mit Galizien und Lodomerien (1772) und Bukowina (1775) ungefähr $272.000 \mathrm{~km}^{2}$.

181 Vocelka 2001, 296. Die erste Volkszählung in Ungarn fand 1784-1787 statt und errechnete 8,56 Millionen Einwohner. Diese Summe wurde durch die späteren Forschungen auf 9,2 Millionen korrigiert, vgl. Wellmann 1979, 296.

$182 \mathrm{Zu}$ den ungarischen Krönungsinsignien gehörten ursprünglich auch die rotseidenen Strümpfe und Bundschuhe, bzw. die weißen Lederhandschuhe und Sandalen, die dem hl. König Stephan zugeschrieben wurden. Diese wurden jedoch 1849 stark beschädigt und letztlich zerstört. Bei der Preßburger Krönung zog Maria Theresia die Sandalen des hl. Stephan jedoch nicht an, weil sie in diesen nicht gehen konnte, ohne umzuknicken, vgl. Hertel 2016a, 116.

183 Bíró Márton Padányi, Regnum decoris et diadema speciei [...], in: Ünnep-napokon [...] mondott külömb-külömbféle sok szép prédikátziók, Győr 1761, 55-56. Nach der Legende wurde die ungarische Königskrone dem hl. Stephan von Engeln gebracht, weshalb sie in der Frühen Neuzeit häufig als „sacra angelica et apostolica corona“ bezeichnet wurde.

184 Nicolaus Benkő, Oratio dum regia Budensi domo perfecta templum aulicum ipso divae Theresiae festo [...] consacraretur dicta, Budae $1769,9$.

185 Gruber 2006, 360. Die „kleine Kapelle“ oder „Privat Capelle der Kayserin“ befand sich am dritten Niveau des Schlosses, vgl. Garms 2010 , 595.

186 Zur Entstehung des Topos vgl. Tüskés / Knapp 2002. 
das Landeswappen, gegen die Osmanen verteidigen. ${ }^{187}$ In der Regierungszeit Maria Theresias erschien auch die Szene der Widmung des Landes an Maria durch König Stephan oft in der Ausstattung jener Räume, die eine besonders wichtige Rolle in der Repräsentation des Königreichs Ungarn und in der Visualisierung der Staatsidee des Regnum Marianum Hungariae spielten. Diese Szene stellte beispielsweise ein Gemälde dar, das sich 1741 im Sitzungssaal des neuerrichteten Landhauses zu Preßburg (in der Lorenzerthorgasse) befand; am Rahmen des Bildes wurde der Doppeladler angebracht, der auf der Brust das Wappen Ungarns trug, welches von den Insignien der zehn Länder der ungarischen Krone umgeben war. ${ }^{188}$ Das von Vinzenz Fischer in höfischem Auftrag gemalte Hochaltarbild der Kapelle der Heiligen Rechten, das 1778 neben der Kapelle des königlichen Palastes in Buda errichtet und 1899 demoliert wurde, stellte ebenfalls die Szene dar, in der König Stephan seine Krone der Muttergottes widmet. ${ }^{189}$ In der oben zitierten Festrede am 20. August 1741 lobte der Propst von Veszprém die besondere Verehrung der hl. Jungfrau als $\mathrm{Pa}$ trona Hungariae durch Maria Theresia: Er betonte, dass die neugekrönte Königin jene Krone erworben habe, die König Stephan der Jungfrau Maria, der Königin des Himmels, anempfohlen habe, wodurch Maria Theresia die Herrscherin des „marianischen Königreichs Ungarn“ geworden sei. Die Ehrfurcht der Regentin vor der Patrona Hungariae wird auch auf einem Gemälde des sogenannten Krönungszyklus im Palais der Ungarischen Botschaft zu Wien visualisiert (vgl. Abb. 125). Das Bild von Franz Messmer und Wenzel Pohl (1768), das den Krönungszug Maria Theresias am Preßburger Hauptplatz thematisiert, stellt den Moment dar, als die Königin vor der Mariensäule, die Leopold I. 1675 zu Ehren der Unbefleckten Jungfrau Maria und Patrona Regni Hungariae errichtet hatte, anhält, um ihre Reverenz zu erweisen. ${ }^{190}$ Es soll hier jedoch bemerkt werden, dass die zeitgenössischen Beschreibungen der Krönungszeremonie diesen Moment des Krönungszugs gar nicht erwähnen. Eine Medaille auf die Huldigung der Stände Siebenbürgens vor Maria Theresia ${ }^{191}$ visualisiert ebenfalls die Ehrfurcht der Herrscherin vor der Patrona Hungariae: Auf der Rückseite kniet die Königin vor Maria und dem apostolischen Doppelkreuz, denen sie ihre Krone zu Füßen legt. ${ }^{192}$ Hier wurde die ikonografische Formel der Darreichung Ungarns durch König Stephan I. an die Himmelskönigin verwendet, als Zeichen dafür, dass Maria Theresia in Ungarn und Siebenbürgen unter dem Schutz der himmlischen Patrona des Landes, der Magna Domina Hungariae, regierte. Die lateinische Inschrift „SUB CRUCE IAM GEMINA GEMINA EST PATRONA MARIA“ betont, dass unter dem Doppelkreuz die zwei Marien - also die Jungfrau und Maria Theresia - als Patroninnen das Land beschützen. Der Gedanke, dass Ungarn unter dem Schutz von zwei Marien steht, wurde auch in der oben zitierten Rede bei dem Fest des hl. Stephan im Jahre 1741 ausgedrückt: Der Redner hat hier zum hl. Stephan gebetet, um die Fürbitte der hl. Jungfrau, „der ersten Patronin und Königin Ungarns mit dem Namen Maria“, zum Herrn zu erflehen, damit das Land unter der „mütterlichen Regierung der Königin Maria II. ein glückliches Schicksal habe“. Der verdoppelte Schutz des Landes durch Gott und Maria, bzw. durch Maria Theresia wurde auch in der Festrede thematisiert, die bei der Konsekration der Wallfahrtkirche Šaštín (Sassin) der schmerzhaften Muttergottes am 15. August 1762 in Anwesenheit des Herrscherpaares gehalten wurde. Der Festredner lobte hier den Glaubenseifer des Kaiserpaares, bzw. betonte den himmlischen Schutz über der Regierung Maria Theresias in Ungarn, der als Konsequenz der (marianischen) Frömmigkeit der Königin gedeutet wurde: „Glückseelige und Gott-gefällige Monarchen, denen der Himmel ein so theures Unterpfand seiner Gnade, seiner Vorsorge und seines Schutzes

187 Jávor 1977.

188 Vgl. Gábor Kolinovics, Nova Ungariae Periodus [...], Buda: Typis Regiae Universitatis 1790, 45.

189 Uxa 1934, 188.

190 AK Maria Theresia Ungarn 1980, Tafel 4; vgl. in diesem Band Linsboth, Krönungsbilder, \#\#.

191 Joseph Wellisch (Münzstätte Karlsburg), 1741.

192 Linsboth 2017a, 49. Die zeremonielle „Treueidablegung“, bzw. Huldigung (solenne homagium in Reginam) fand in Hermannstadt am 21. Februar 1741, am Tag der Eröffnung des Siebenbürgischen Landtags statt, vgl. Trócsányi 1985, 12. (Der Katalog Schau- und Denkmünzen behauptet irrtümlich, dass die Huldigung gleich nach der Preßburger Krönung Maria Theresias, im Sommer 1741 stattfand, vgl. Schau- und Denkmünzen 1782, 32-33, Nr. XXV) Der Entwurf der Medaille stammte vom Calvinisten (!) Baron Johann Lázár von Gyalakuta (1703-1772), Assessor an der siebenbürgischen königlichen Tafel, der 1742 auch in einer kleinen Druckschrift die Bedeutung des Bildes erklärte: vgl. Johann Lázár von Gyalakuta, Descriptio et explicatio imaginum inscultarum numismati [...], Wien 1742. Vom Flugblatt ist jedoch kein erhaltenes Exemplar bekannt. Es ist bemerkenswert, dass trotz der konfessionellen Spaltung die protestantische Gemeinde Siebenbürgens ihre Loyalität zur Königin auch nach dem Tod Maria Theresias zum Ausdruck brachte. Im Jänner 1781 wurde in der evangelischen Stadtpfarrkirche von Hermannstadt ein Trauergerüst errichtet; gleichzeitig wurden in mehreren Reformierten Kirchen Siebenbürgens Trauerreden über die „Herrin des Ungarischen Sions“ und „neue Deborah“ gehalten, die auch im Druck erschienen, vgl. O. A., A Magyar Sionnak Felséges Asszonya [...], Kolozsvár 1782. 
geschenket hat! [...] Allergnädigste Frau! Euer Majestät allermildestes und Erbarmungs-volles Mutter-Herz, welches Dieselben gegen alle Arme, Preßhafte, Bedrangte und Nothleidende tragen [...] dessen rechte Hand [d. h. Gottes] auch nur kürzlich eine so wichtige Beränderung veranlasset hat, daraus wir Sonnen-klar sehen, und ungezweifelt schliessen können, daß sein Auge über Euer Majestät, dero Durchläuchtigstes Erzhaus, und gesamte Staaten unaufhörlich wache, und daß Dero Apostolisches Königreich Ungarn unter dem Schutz seiner grossen Frau, und unter der Regierung Euer Majestät vor allen feindlichen Anfällen gesichert seyn könne. “193

Die Regierung Maria Theresias in Ungarn, im „Königreich des heiligen Stephan“, ist in diesen Huldigungsschriften und in den zeitgenössischen Prädikationen und Lobreden als Blütezeit, als Zeitalter der Konsolidierung und Konjunktur nach der anderthalb Jahrhunderte langen osmanischen Besetzung charakterisiert worden. So pries etwa Zsigmond Keglevich, Domherr des Graner Domkapitels 1777 in Tyrnau, am Festtag der hl. Theresia, also am Namenstag Maria Theresias, die Regierung der Königin folgenderweise: „Ist wohl Ungarn von Urzeiten her mit einem so langen, so vortheilhaften Frieden beseliget worden, wie der ist, dessen wir uns unter der segenvollen Regierung dieser Großen Fürstinn rühmen können? Und wie viele andere Vortheile sind allenthalben dem gemeinen Besten zugewachsen? Man mag nun den Ruhm der Waffen, oder die den Wissenschaften und Künsten gestifteten Denkmaale, oder den Vorzug betrachten, mit welchem Tugend und Verdienst vor dem königlichen Throne geadelt wird, so kann man behaupten, daß unter dem glänzenden Zepter Marien Theresien Ungarn die Augen aller auswärtigen Völker auf sich gezogen hat. ${ }^{\text {199 }}$

Im 17. Jahrhundert wurde das Land politisch dreigeteilt (nämlich in das Königreich Ungarn, das Fürstentum Siebenbürgen und die osmanisch besetzten Gebiete) und auch die Verbreitung des Protestantismus führte zu schweren religiösen Konflikten. Diese zweifache Trennung des Landes wurde im Laufe des 18. Jahrhunderts im Wesentlichen behoben. In der Ära Maria Theresias war das Königreich, das mit der Rückeroberung der osmanisch besetzten Gebiete um 1700 wiedervereinigt wurde, tatsächlich durch eine friedliche Entwicklung geprägt, und im Laufe des 18. Jahrhunderts wurde der entvölkerte, früher osmanisch besetzte mittlere Teil Ungarns wieder besiedelt, wodurch sich die Bevölkerungszahl ${ }^{195}$ wie auch die Zahl der Menschen mit römisch-katholischem Glauben verdoppelten. Parallel dazu wurde die institutionelle und wirtschaftliche Infrastruktur des Landes konsolidiert.

Im 18. Jahrhundert bestimmte diese grundsätzlich positive Perzeption die literarischen, politischen und künstlerischen Bilder und Konzepte Ungarns im In- wie im Ausland. Sowohl das Selbstbild der Ungarn als auch die Vorstellung von Ungarn in Europa, das image of the other, wurde zu dieser Zeit neugeprägt. Dieses inhaltlich positive Bild Ungarns, welches das Wohlergehen des Landes betonte, ist ein Novum in den Bildmedien des 18. Jahrhunderts, insbesondere in der maria-theresianischen Ära. In den vorigen zwei Jahrhunderten wurde Ungarn nämlich meistens im Zusammenhang mit den Türkenkriegen allegorisiert. Zu dieser Zeit war es ein bekanntes Konzept, die historische Rolle Ungarns und seine Lage in Europa als „Bollwerk des Christentums" gegen die Osmanen darzustellen. ${ }^{196}$ Der Topos Hungaria est Propugnaculum Christianitatis war im 16. und 17. Jahrhundert nicht nur in der Rhetorik, sondern auch in den Bildmedien der Zeit weit verbreitet. Ein effektvolles Beispiel dafür ist ein anonymer Kupferstich aus dem 17. Jahrhundert, vermutlich aus der Tyrnauer Universitätsdruckerei, ${ }^{197}$ in dem Ungarn als Festung und vorgesetzte Bastei mit der Ansicht der römischen Engelsburg im Hintergrund dargestellt wird. ${ }^{198}$

Diese Darstellungen, die den Heldenmut und die Kampftugenden der Ungarn thematisieren, könnten in den 1740er-Jahren als Vorbild für jene Grafiken gedient haben, welche die Beteiligung von ungarischen Soldaten am Österreichischen Erbfolgekrieg propagierten und die Unterstützung Maria Theresias durch den ungarischen Adel betonten. In diesem Jahrzehnt wurde der Beistand der Ungarn besonders häufig auf den Werken des Augsburger Kupferstechers Gottfried Bernhard Götz thematisiert.

193 Samuel Engelmayer, Lob- und Lehr-Rede gehalten bey Gelegenheit der feyerlichen Überbringung der schmerzhaften Mutter Gottes von Schossberg [...] in die neuerbaute [...] Kirche des Ordens des Heil. Pauli, Ofen 1762, 7. Zum herrscherlichen Mäzenatentum des Gnadenorts vgl. Serfőző 2009.

194 Siegmund Keglevics, Dankrede auf Ihre kaiserl. königl. apostolische Majestät Maria Theresia [...], Tyrnau 1777, 6.

195 Die Bevölkerungszahl Ungarns um 1720 wird auf vier Millionen geschätzt, vgl. Kovacsics 1963, 435.

196 Der Topos erschien bereits im 15. Jahrhundert, setzte sich aber nach der Schlacht bei Mohács (1526) allgemein durch, vgl. Imre 2004; Etényi 2014; Lénárt 2016, 161-182. Zum westeuropäischen Bild Ungarns im Mittelalter vgl. Csukovits 2015.

197 Der Stich in der Größe von 24,5 x 19,5 cm erschien wahrscheinlich als Illustration einer Druckschrift, die jedoch unter den lateinischen Publikationen der Tyrnauer Druckerei aus dem 17. Jahrhundert nicht identifiziert werden konnte.

198 AK Magyarország 2004, 19; Bubryák 2014, Abb. 2. 


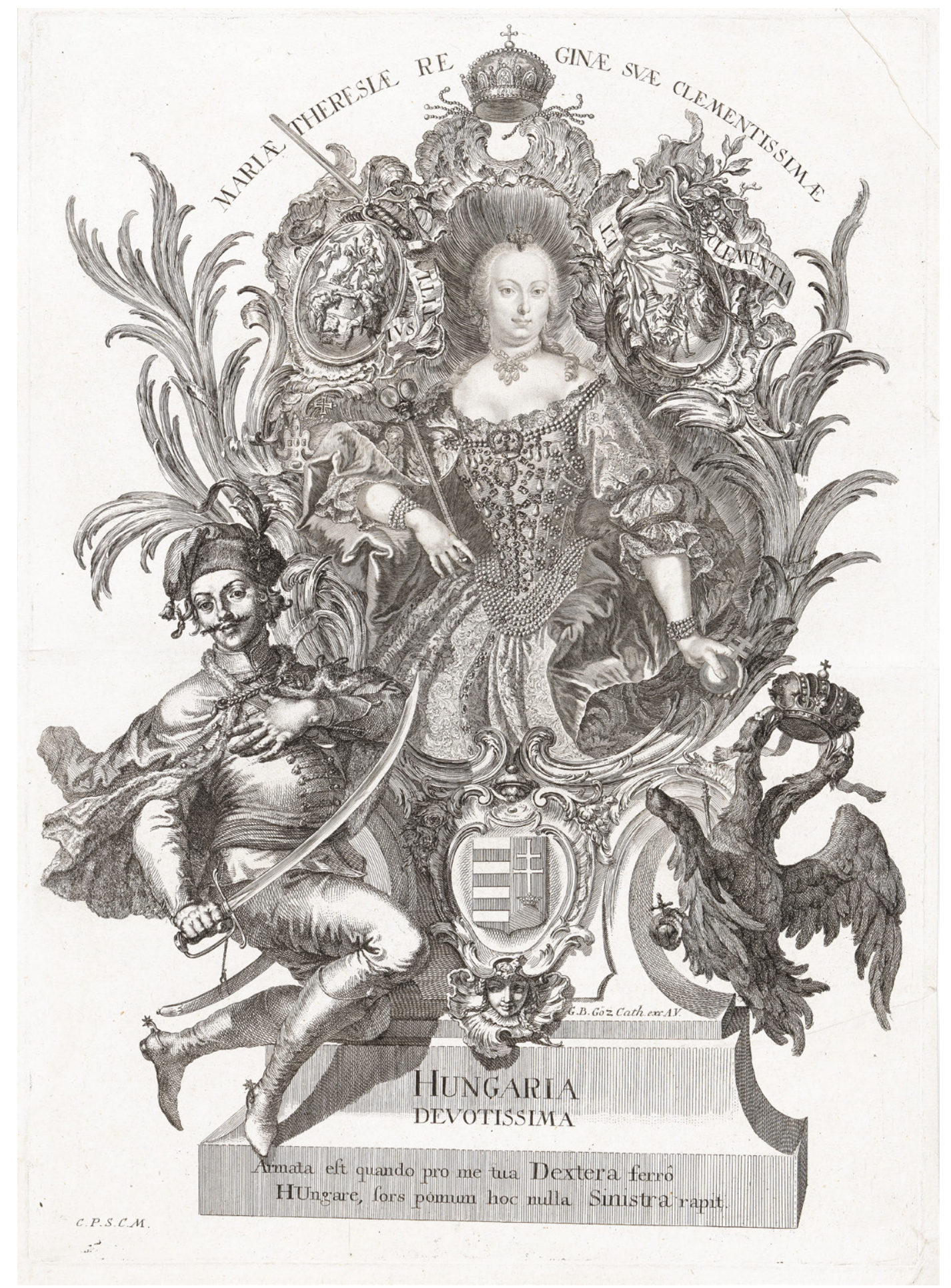

Abb. 132: Gottfried Bernhard Götz, Verherrlichung Maria Theresias als Königin von Ungarn, 1743 (Wien Museum, 179425)

Das effektvollste darunter ist ein Stich im Folioformat, der die Huldigung Ungarns vor Maria Theresia thematisiert (Abb. 132). Hier ist Maria Theresia als Königin von Ungarn im ungarischen Krönungsornat abgebildet, wobei ihr links ein kniender ungarischer Husar huldigt; in seiner Rechten hält er einen Säbel, die andere Hand ruht als Symbol der Treue auf seinem Herzen. Auf dem Postament in der Mitte ist die Inschrift „HUNGARIA DEVOTISSIMA“(„Das 


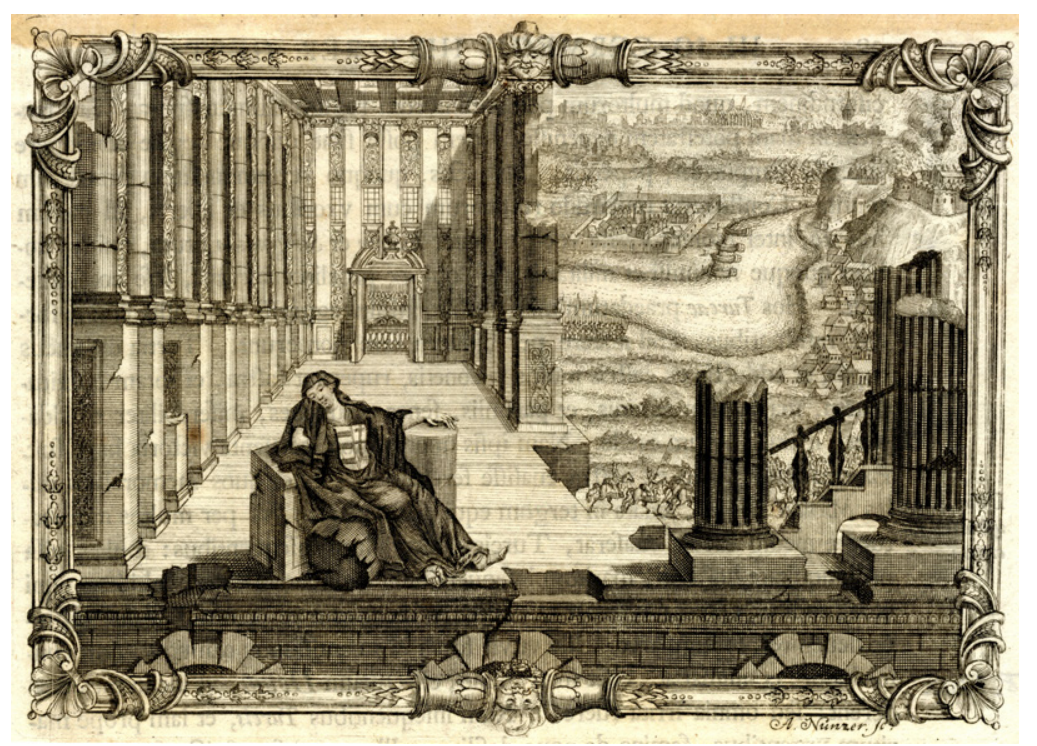

Abb. 133: Andreas Nunzer, Schlafende Hungaria, 1746 (MNM, 583702)

treueste Ungarn“) zu lesen, als Hinweis auf die ungarischen Stände, die ihr Leben und Blut der Königin anboten. ${ }^{199}$

Im Zusammenhang mit den Türkenkriegen wurde Ungarn in den bildlichen Medien oft auch als Opfer der Geschichte präsentiert. In dieser Rolle erscheint die weibliche Personifikation Ungarns zum Beispiel auf einem Holzschnitt in einer Druckschrift von 1581. ${ }^{200}$ Hungaria wird hier von den Osmanen gemartert, während Germania, an der linken Seite, ihr zu Hilfe eilt. Im 17. Jahrhundert ist das unter der Herrschaft und den Angriffen der Osmanen leidende und sein eigenes Schicksal beklagende Ungarn (Querela Hungariae) zu einem visuellen Topos geworden. ${ }^{201}$ So erscheint auf einem Titelblatt von 1665 Hungaria mit einem Trauer-Tuch auf dem Kopf, wie sie sich und die Völker Europas mit Schwert und Schild gegen die Osmanen verteidigt. ${ }^{202}$ Die allegorische Figur der trauenden Hungaria bestand in den Bildmedien bis zum Endes des 18. Jahrhunderts fort: Eine eindrucksvolle Visualisierung des Themas ist eine Illustration aus einer ungarischen Sammlung von Geschichtsquellen, die 1746 unter dem Titel Scriptores Rerum Hungaricarum erschien und von dem Bibliothekar der kaiserlichen Hofbibliothek herausgegeben wurde. ${ }^{203}$ Ein Bericht über die Türkenkriege des 16. Jahrhunderts wurde mit einem Kupferstich von dem in Nürnberg tätigen Andreas Nunzer illustriert, wobei Ungarn als schlafende Nymphe, in der Gestalt einer schwarzgekleideten Frau, dargestellt wird, die in einem imposanten, jedoch zum Teil zerstörten Palast schläft (Abb. 133). Im Hintergrund ist die Rückeroberung Ofens 1686 abgebildet. Es ist nicht eindeutig, ob die Schlachtszene Realität oder Traum der schlafenden Hungaria ist, jedoch erscheint der Palast hier deutlich als Zeuge der glorreichen Vergangenheit des Landes und als Symbol des zu rekonstruierenden Königreichs, während der Schlaf auf die kommende Erweckung Ungarns hinweist. In der Komposition kommt also auch die Idee der Wiederinstandsetzung und Wiedergeburt des Königreichs Ungarn zum Ausdruck. Das Bild ist eines der wenigen Beispiele aus der Zeit, welches das Regnum Hungaricum als eigenes Thema, unabhängig von Maria Theresia, darstellt.

Der Gedanke der Erneuerung Ungarns spiegelt sich auch auf dem Titelkupfer der obenerwähnten Sammlung von Geschichtsquellen wider: Hier ist Chronos dargestellt, wie er den Titel des Werks auf eine Steinplatte graviert. ${ }^{204}$ Im Hintergrund wird ein symbolisches Bauwerk von Putti errichtet. Auf einem Altar in der zentralen Nische liegt die Stephanskrone. An dem Altar ist ebenfalls die Devise Maria Theresias, „Iustitia et Clementia“, zu lesen. Das Bauwerk lässt sich also als Tempel des Königreichs Ungarn und zugleich als Reich der Stephanskrone interpretieren, das unter der Regierung Maria Theresias, im Zeichen ihres Wahlspruchs mit „Gerechtigkeit und Milde“, wiederaufgebaut wird.

199 AK Maria Theresia Ungarn 1980, 177, Nr. 107; Szilárdfy 2009.

200 Martin Schrot, Wappenbuch des Heiligen Römischen Reichs und allgemeiner Christenheit in Europa, München: Berg 1581, 13, vgl. Marosi 2009, 130-131.

201 Imre 1999.

202 Hieronymus Oertel, Ortelius redivivus et continuatus oder der Ungarischen Kriegs-Empörungen, Nürnberg: Paul Fürst 1665.

203 Johann Georg Schwandtner, Scriptores rerum Hungaricarum, Bd. 2, Viennae 1746, 383, vgl. Marosi 2009, 162.

204 Ebd., 163. 


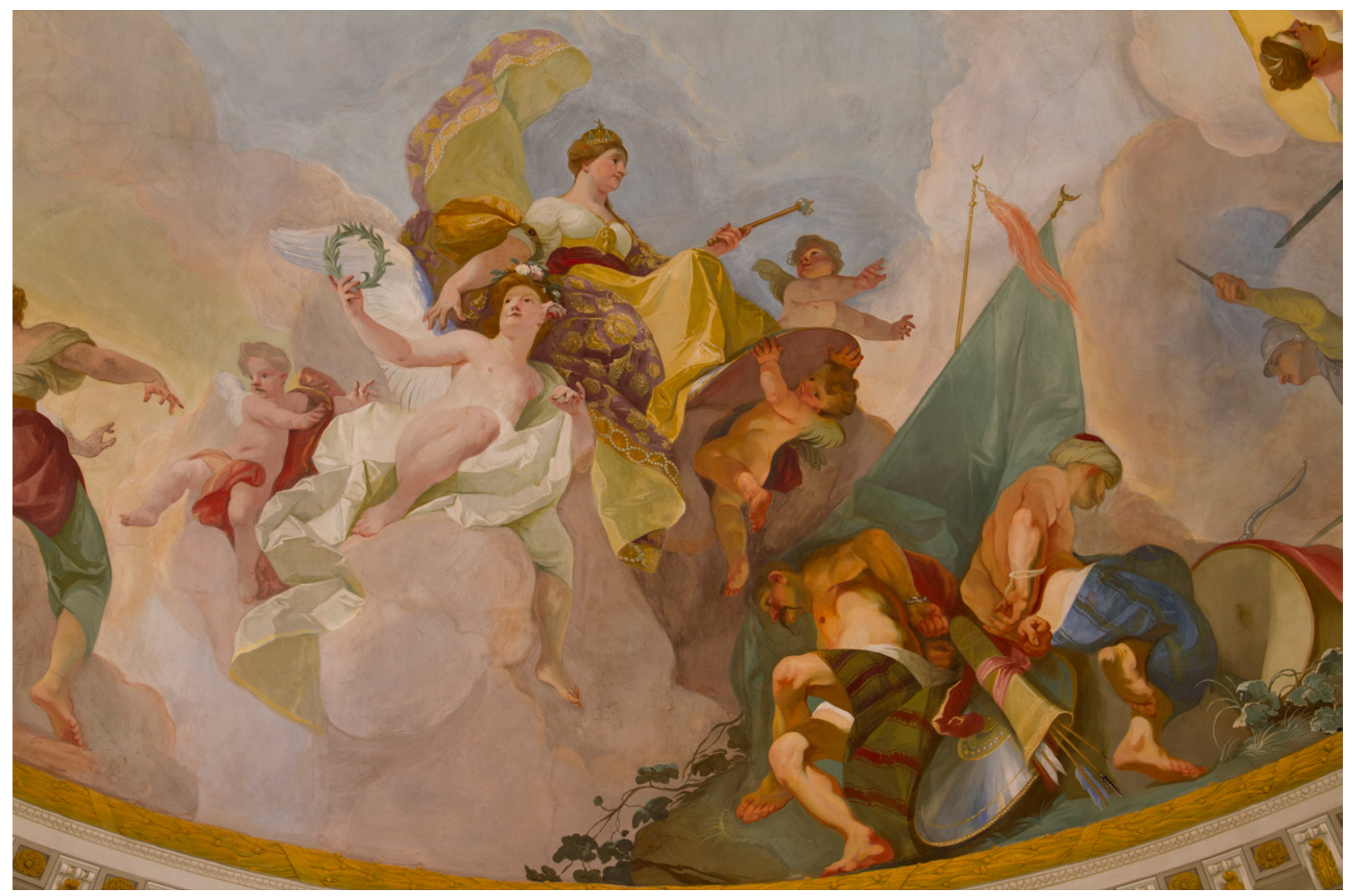

Abb. 134: Franz Anton Maulbertsch, Allegorie des „erlösten Ungarn“, Detail des Deckenfreskos im Riesensaal der Hofburg in Innsbruck, 1775 (ÖAW, IKM)

Am Ende des 17. Jahrhunderts, als der Triumph der Habsburgermonarchie über das Osmanische Reich in den visuellen Medien thematisiert wurde, erscheinen neue Formen der Personifikation der Hungaria, welche die Befreiung des Landes verherrlichen: Auf einer Medaille auf die Rückeroberung Ofens (1686) kniet die weibliche Figur der Hungaria als Versinnbildlichung des befreiten Königreichs Ungarn vor Leopold I. Der Kaiser steht in der Rüstung eines antiken Imperators und wird von Viktoria gekrönt, als er auf den nach unten zeigenden türkischen Halbmond tritt und zugleich mit seinem Zepter die Personifikation Ungarns berührt. Begleitet wird diese Geste durch die Inschrift, die dem Habsburger als Restitutor Vngariae huldigt. ${ }^{205}$ Im Hintergrund erscheint auch die Personifikation der katholischen Kirche Ungarns mit Doppelkreuz. Diese politische Programmatik konnte zur Regierungszeit Karls VI. und Maria Theresias durch die kraftvolle Durchsetzung der Rekatholisierung, bzw. der katholischen Konfessionalisierung, tatsächlich erfolgreich realisiert werden.
Diese bildliche Tradition, welche die Rolle der Habsburger bei der Befreiung Ungarns verherrlicht, hat auch die Darstellung Ungarns auf dem mittleren Deckenfeld im Riesensaal der Innsbrucker Hofburg beeinflusst (Abb. 134). Das Deckenfresko von Franz Anton Maulbertsch (1775) hat die Allegorie auf die Verbindung der Häuser Österreich und Lothringen zum Thema: ${ }^{206} \mathrm{Im}$ Rahmen des reichen bildlichen Programms weist die Personifikation Ungarns auf die Türkenkriege hin. Der Sieg über das Osmanische Reich wird hier also als gemeinsame glorreiche Tat der Habsburger und der Herzöge von Lothringen präsentiert. Die Personifikation des „erlösten Ungarn“ erscheint als Pendant zum „befreiten Wien“. Unten, in den dreieckigen Grisaille-Bildfeldern ist der Entsatz von Wien, bzw. die Rückeroberung von Ofen dargestellt. Hungaria erscheint als prächtig bunt gekleidete Frau mit der Stephanskrone auf dem Haupt, wie sie über die besiegten Osmanen triumphiert, die als Gefangene im Vordergrund sitzend dargestellt sind. Neben ihr hält ein rosenbekränzter, geflü-

205 Georg Hautsch (Medailleur), Nürnberg. Inschrift: „LEOPOLDO VLTORE RESVRGES“. Die Rückseite zeigt die von Reichstruppen eroberte Stadt Ofen, vgl. Galavics 1986, Abb. 76; Schumann 2004, 328, Abb. 35.

206 Haberditzl 2006, 289; Telesko 2015b. 
gelter Genius den Siegeskranz empor. Rechts ist die Personifikation des Heldenmuts als Krieger abgebildet, der vom Frieden mit Lorbeer bekränzt wird. Diese Figur weist wohl auch auf Herzog Karl V. von Lothringen, den Großvater Kaiser Franz I. Stephans, hin, der als kaiserlicher Feldherr bei der Eroberung von Ofen 1686 eine entscheidende Rolle gespielt hatte.

Auf einem anderen monumentalen Freskowerk der Ära, und zwar auf dem mittleren Deckenfresko in der Großen Galerie von Schloss Schönbrunn, wurde ein anderer Topos in Bezug auf Ungarn visualisiert, nämlich die Fruchtbarkeit des Landes. Das Fresko von Gregorio Guglielmi (1760) thematisiert das Wohlergehen der Monarchia Austriaca. ${ }^{207}$ Die im Zentrum angebrachte allegorische Figurengruppe mit dem Herrscherpaar ist von Personifikationen der Kronländer der Habsburgermonarchie und ihren typischen Produkten und Erzeugnissen umgeben. Am prominentesten Platz, rechts unterhalb der Mittelgruppe, erscheint das Königreich Ungarn, personifiziert in einer Frauengestalt, welche die Stephanskrone und das ungarische königliche Zepter hält (vgl. Taf. 26). Rechts von ihr schwebt eine weibliche Figur in goldenem Mantel mit dem Doppelkreuz, dem heraldischen Symbol Ungarns, in der Hand. Die weiteren Figuren der Gruppe weisen auf die Wirtschaft des Landes hin und unterstreichen dessen landwirtschaftlichen Charakter. Eine nackte Frauenfigur mit Weizengarbe und Blumen repräsentiert die ungarische Landwirtschaft. Neben ihr ist ein Flussgott mit Fisch zu sehen, als Personifikation der Fischzucht oder der Donau, bzw. der Theiß. In der irdischen Zone sind Hirten und ihre Tiere als Versinnbildlichung der ungarischen Viehzucht zu sehen. Neben Getreide war Vieh ein weiteres wichtiges Exportgut Ungarns in der Frühen
Neuzeit. Der in der Mitte stehende Gulyás trägt die traditionelle Kleidung der Viehhirten, eine aus Schafpelz gefertigte Schaube $(s u b a){ }^{208}$

In der Mittelachse des Bildfelds schwebt Merkur, der mit seinem Caduceus auf die Personifikation Ungarns deutet. Seine Figur weist darauf hin, dass in der Förderung des Handels die materielle Grundlage des Gemeinwohls gesehen wurde. Unter ihm ist eine Gruppe von Bergmännern mit verschiedenen Werkzeugen des Bergbaus dargestellt. Rechts ist ein aus der Erde emporsteigender Bergmann zu sehen, der einen großen Goldklumpen hält. Links sehen wir eine Münzprägemaschine, daneben wiegt ein Mann Goldmünzen ab. Diese Figuren weisen wahrscheinlich auf den ungarischen Bergbau und das Münzwesen hin, ${ }^{209}$ da die Erträge der Goldgruben von Siebenbürgen, bzw. der Silber- und Kupferbergwerke von Oberungarn nicht nur im Habsburgerreich, sondern in ganz Europa am bedeutendsten waren, ${ }^{210}$ und so als wichtige Einnahmequelle des Hofs dienten. Die Einkünfte aus den ungarischen Bergwerken wurden etwa 1770 auf 1,2 Millionen Gulden geschätzt. ${ }^{211}$ Die herausragende Bedeutung der oberungarischen königlichen Bergwerke wird nicht zuletzt dadurch deutlich, dass sowohl Kaiser Franz Stephan als auch Joseph II. diese 1751, bzw. 1764 besichtigten. ${ }^{212}$ Bei letzterer Gelegenheit erhielt Erzherzog Joseph einen Handstein mit detailreicher Darstellung des dortigen Bergwerks von der Stadt Kremnitz als Geschenk. ${ }^{213}$

Die Inszenierung Ungarns als fruchtbares Land, wie sie auch im Schönbrunner Deckenfresko präsent ist, ist eine für das 18. Jahrhundert geläufige Stereotypie, die den Topos des von den Osmanen geplagten Ungarn zur Regierungszeit Karls VI. ersetzt hatte. ${ }^{214}$ Der Gedanke der Fertilität

207 Garas 1963; Telesko 2016b.

208 Seine hoher Hut (csákós kunsüveg) ist jedoch typisch für den Rosshirten in Kumanien (Kunság) in der Tiefebene, vgl. Bikessy-Heimbucher 1816; Bikessy-Heimbucher 1820, Tafel 20.

209 Werner Telesko hält es für fraglich, ob diese Figuren inhaltlich mit dem Königreich Ungarn zu verbinden sind, vgl. Telesko $2016 \mathrm{~b}, 89$. Nachdem aber auf einer Landkarte Ungarns von 1769 ebenfalls der Bergbau und die Landwirtschaft als zwei fundamentale Wirtschaftszweige des Königreichs dargestellt wurden (Ignác Müller, Mappa Geographica novissima Regni Hungariae, Viennae 1769), scheint diese Identifizierung auf dem Deckenfresko in Schönbrunn plausibel.

210 Rádóczy 1980, 10.

211 Dickson 1980.

212 Kulcsár 1999; Kulcsár 2005.

213 KHM, KK 4146.

214 Ungarn war im 18. Jahrhundert nach der langen osmanischen Besetzung wirtschaftlich und gesellschaftlich etwas unterentwickelt. Das städtische Bürgertum machte weniger als $10 \%$ der Bevölkerung aus. Der Anteil des Adels betrug etwa 8 \%, während die große Mehrheit der Bevölkerung (über 80 \%) dem Bauerntum angehörte, das in über 14.000 Dörfern und Meiereien auf dem Land lebte (vgl. Fónagy 2001). Diese überwiegend rurale Gesellschaft war durch eine grundlegend landwirtschaftliche Produktion geprägt. Dieser Umstand spiegelte sich auch in der ungarischen Kontribution zum Staatsbudget wider: Der Anteil der Einkünfte aus den ungarischen Ländern von den Gesamteinnahmen der Regierung betrug nur zirka 25-30 \% (etwa 16 Millionen von den 62 Millionen Gulden Gesamteinnahmen), vgl. Dickson 1980, 93. Das Land wurde jedoch wegen seiner guten natürlichen Gegebenheiten, seiner fruchtbaren Äcker, Weiden und Wälder mit gutem Recht die „Speisekammer“ der Habsburgermonarchie und Europas genannt. 
Ungarns als literarischer Topos stammt aus dem Spätmittelalter; ${ }^{215}$ die visuellen Darstellungen des Themas erscheinen jedoch erst am Anfang des 18. Jahrhunderts.

Inhaltlich eng verbunden mit der Komposition Guglielmis ist ein Kupferstich der Gebrüder Schmutzer aus dem Jahre 1735, der das unter der Herrschaft Kaiser Karls VI. erblühende Ungarn zum Thema hat und als Vorsatzblatt zum Werk des lutherischen Theologen und Geschichtsschreibers Matthias Bél erschien. ${ }^{216}$ Die zahlreichen motivischen Übereinstimmungen mit dem Fresko Guglielmis weisen darauf hin, dass er den Kupferstich als Vorbild verwendet haben könnte.

Als besonders reiches Land wird Ungarn auch auf einem anderen Kupferstich dargestellt, wobei die Fruchtbarkeit des Königreichs durch die weibliche Figur mit dem Füllhorn in der Mitte der Komposition versinnbildlicht wird. Das Blatt ist Teil einer 1736 in Augsburg erschienenen Serie, die eine stereotypische Nationalcharakterologie der Völker Europas liefert. ${ }^{217}$ Eine ähnliche Illustration befindet sich auch in den obenerwähnten Scriptores rerum Hungaricarum, die eine wirkungsvolle Visualisierung der Verwaltung Ungarns liefern. Die Regierungsform des Landes war eine sogenannte Stände-Vertretungsmonarchie, das heißt, dass die geistlichen und weltlichen Stände Ungarns in der Legislative neben dem König einen gleichberechtigten Faktor bildeten. König und Königreich, repräsentiert durch die „Nation“, waren also gleichwertige Partner, überhöht von der hl. Stephanskrone, einander verpflichtet durch den Akt der Krönung. Diese Staatsidee wurde in unterschiedlicher Weise durch Kunst, Bildmedien und Zeremoniell der Epoche verdeutlicht; darunter auch durch den Kupferstich, der Maria Theresia auf dem Thron darstellt, umgeben von den ungarischen geistlichen und weltlichen Ständen. ${ }^{218}$
Eine andere Illustration aus demselben Werk zeigt die ungarische Königskrone, umgeben von den Fahnen der zehn historischen Länder der hl. Stephanskrone, das heißt mit den Bannern von Ungarn, Kroatien, Dalmatien, Slawonien, Lodomerien, Serbien, Bosnien, Bulgarien, Galizien und Kumanien. ${ }^{219}$ (Die letzteren sind als Anspruchstitel zu verstehen, die seit dem Mittelalter Teil der ungarischen Königstitulatur waren.) Die zehn Landesfahnen repräsentieren hier also die Glorie des Reichs der Stephanskrone, zugleich aber auch die territoriale Integrität und Einheit des Königreichs, die am Anfang des 18. Jahrhunderts wiederhergestellt wurde. Dieser Gedanke wird auf dem Titelblatt einer topografischen Beschreibung Ungarns von 1718 deutlich zum Ausdruck gebracht: Hier sitzt die Personifikation der Hungaria am Fuß eines pyramidenförmigen Triumphdenkmals, von Kriegstrophäen und den zehn Landesfahnen umgeben. ${ }^{220}$

Die zehn Landesfahnen des Reichs der Stephanskrone hatten in der Frühen Neuzeit auch bei der ungarischen Krönungszeremonie eine Rolle gespielt, wo sie von den Mitgliedern der prominentesten adeligen Familien getragen wurden. ${ }^{221}$ Sie sind auf einem Kupferstich der Krönung von Leopold I. in Preßburg (1655) gut erkennbar. ${ }^{222}$ Diese Fahnen waren auch bei der Preßburger Krönung Maria Theresias präsent, wie sie auf einem Gemälde des Krönungszyklus im Palais der ehemaligen Ungarischen Hofkanzlei in der Bankgasse, an der linken Seite des Chors des Martinsdoms, $\mathrm{zu}$ sehen sind (vgl. Taf. 19). ${ }^{223}$

Die Einheit der ungarischen Krone mit ihren Ländern und des Königs mit den Ständen wird ebenfalls auf einem Kupferstich betont, der als Frontispiz zu einem Atlas von Ungarn 1751 erschien. ${ }^{224}$ Aber auch Hinweise auf die friedliche Entwicklung des Landes fehlen nicht: Im Hintergrund sehen wir Szenen aus Landwirtschaft und Viehzucht,

215 Vgl. Tarnai 1969, 43-44.

216 Matthias Bél, Notitia Hungariae Novae Historico Geographica, Bd. 1, Wien 1735, vgl. AK Prinz Eugen 1986, Nr. 2.8. Kaiser Karl hat dem Verfasser für sein Werk eine jährliche Besoldung von 600 Gulden bestimmt, vgl. Ebner 1906, Bd. 2, 15.

217 Hungarus, Blatt 8 aus der Völkertafel: Laconicum Europae Speculum: Kleiner Spiegel Europas, Augsburg 1736. Papier, Kupferstich, Radierung; 36,5 x 41,9 cm (Platte); Zeichner: Paul Decker; Stecher: Christian Friedrich Lottes; Herausgeber: Martin Engelbrecht, vgl. AK Batthyányak 2005, Nr. VI.20.

218 Johann Georg Schwandtner, Scriptores rerum Hungaricarum, Bd. 2, Viennae 1746, 607.

219 Ebd., 435.

220 Michael Bombardius / Johannes Tersztyánszky, Topographia magni regni Hungariae [...], Wien: Voigt 1718. Das Titelblatt der zweiten, ergänzten Auflage des Werks, das 1750 erschien, stellt Maria Theresia auf dem Thron Ungarns dar. Die zehn Landeswappen, ergänzt um das Wappen Siebenbürgens, erscheinen hier als Muster des Fußbodens. Vor der Königin steht Erzherzog Joseph in ungarische Adelstracht gekleidet, der von Herkules und Minerva zum Thron begleitet wird. Als Inspirationsquelle dürfte hier wohl das Titelblatt des Geometriebuchs für Joseph I. gedient haben, vgl. Burckhard von Birckenstein, Ertzherzogliche Handgriffe [...], Wien 1686.

221 Pálffy 2009, 121; Pálffy 2010.

222 Polleroß 2010, Abb. 8.

223 AK Maria Theresia Schönbrunn 2017, 31 (Abb.). Die zehn Fahnen sind auch auf dem Gemälde (an der rechten Seite) dargestellt, das die Zeremonie des Ritterschlags in der Franziskanerkirche darstellt, vgl. Abb. 123.

224 Titelkupfer zu: János Tomka-Szászky, Parvus Atlas Hungariae, Preßburg 1751, vgl. AK Maria Theresia Ungarn 1980, 164, Nr. 44; Rózsa 1998, Nr. 29. 
im Vordergrund einen Flussgott und Abundantia mit dem Füllhorn. Die vier Männer in ungarischen und kroatischen Kleidung repräsentieren die Völker des Landes als Zeichen dafür, dass im 18. Jahrhundert das Königreich Ungarn sowohl in ethnischer und sprachlicher als auch in konfessioneller Hinsicht äußerst vielfältig war. Um 1780 waren von den neun Millionen Einwohnern der Länder der ungarischen Krone nur 40 \%, d. h. 3,6 Millionen Ungarn, die anderen $60 \%$ setzten sich aus verschiedenen Nationalitäten zusammen (darunter rumänische, slowakische, deutsche und weitere Muttersprachler). ${ }^{225}$

Zum Schluss sei hier ein „Trauerwerk“ aus dem Jahre 1780 von Jakob Matthias Schmutzer vorgestellt. ${ }^{226}$ Die in Zusammenhang mit den Türkenkriegen entstandene Figur der trauernden Hungaria wurde hier auf den Tod der Königin aktualisiert und adaptiert. Die Personifikation der trauernden Hungaria am Doppelsarkophag des Herrscherpaars in der Kapuzinergruft könnte hier wohl als Inspirationsquelle gedient haben. Das Blatt im Folioformat, das Pannoniens Trauer am Grabe Maria Theresias darstellt, zeigt ein fiktives Grabmal der Regentin, neben dem Hungaria den Verlust der Monarchin beweint. Die lateinische Inschrift auf dem Grabmal fasst die wichtigsten Taten Maria Theresias zusammen, darunter die Ereignisse ihrer Regierung in Ungarn. In einer zeitgenössischen deutschen Übersetzung lautet es: „Ungarn hat sich in der Fortdauer des Friedens unter meiner Regierung von den hundertjährigen Drangsalen des Krieges wieder erholt. Ich habe es durch Wiederbringung alter Reiche erweitert, und Themis mit den verscheuchten Musen in seinen Schoos zurück geführt. "227

Gemäß der Signatur wurde die Komposition von Baron Johann Schilson entworfen, der wirklicher Geheimer Rat der ungarischen Hofkammer und königlicher Kommissär der königlichen Freistadt Ödenburg war und sich als aristokratischer Dilettant für Architektur interessierte. ${ }^{228}$ Entsprechend des Berichts des Preßburger Ungarischen Magazins - das Epigramm verfasste Lázár Somssich, Sekretär der ungarischen Statthalterei, - wurden die Kosten der Platte von Baron László Orczy getragen. Der Stich ist also eines der wenigen Beispiele, bei denen die Akteure des Herstellungsprozesses genau bekannt sind.

Hungaria betrauerte den Tod Maria Theresias aus gutem Grund. Die Regierungszeit der Königin wurde nicht nur mittels sprachlicher und bildlicher Rhetorik auf poetische Weise als Blütezeit charakterisiert. Die Epoche Maria Theresias gilt auch in der historischen Erinnerung als eine Art goldenes Zeitalter in der Geschichte Ungarns. Mit dem Namen Maria Theresia sind im Urteil der modernen Geschichtsschreibung die Begriffe der friedlichen Entwicklung und Konjunktur verbunden. Ein unanfechtbarer Verdienst der Königin ist es, dass sie das Land nach der langen osmanischen Besetzung erfolgreich konsolidieren konnte.

225 Darunter gab es $16 \%$ rumänische, jeweils $10 \%$ slowakische, kroatische und deutsche, $6 \%$ serbische, und jeweils $3 \%$ slowenische und russische Muttersprachler. 55 \% der Bevölkerung gehörte dem römisch-katholischen Glauben, 25 \% den protestantischen Konfessionen, weitere $20 \%$ den griechisch-katholischen und orthodoxen Kirchen an; die Israeliten repräsentierten über 1\%, vgl. Kosáry 1980, 62.

226 AK Maria Theresia Ungarn 1980, 239, Nr. 487 (Gizella Cenner-Wilhelmb).

227 Ungarisches Magazin [Preßburg: Anton Löwe], 2 (1782), 3. Zitiert nach: Papp 2000, 121.

228 Fallenbüchl 1989, 82. 